\title{
@creative
}

ISSN 1855-3966 (printed edn.), ISSN 1855-3974 (electronic edn.)

ARS MATHEMATICA CONTEMPORANEA 16 (2019) 67-79

https://doi.org/10.26493/1855-3974.1546.c5e

(Also available at http://amc-journal.eu)

\section{On chromatic indices of finite affine spaces*}

\author{
Gabriela Araujo-Pardo \\ Instituto de Matemáticas, Universidad Nacional Autónoma de México, \\ Ciudad Universitaria, 04510, México City, México \\ György Kiss
}

Department of Geometry and MTA-ELTE Geometric and Algebraic Combinatorics Research Group, Eötvös Loránd University, H-1117 Budapest, Pázmány s. 1/c, Hungary and FAMNIT, University of Primorska, 6000 Koper, Glagoljaška 8, Slovenia

\section{Christian Rubio-Montiel}

Department of Algebra, Comenius University, Mlynska dolina, 84248, Bratislava, Slovakia and División de Matemáticas e Ingeniería, FES Acatlán, Universidad Nacional Autónoma de México, 53150 Naucalpan, México

\section{Adrián Vázquez-Ávila \\ Subdirección de Ingeniería y Posgrado, Universidad Aeronáutica en Querétaro, Parque Aeroespacial Querétaro, 76270, Querétaro, México}

Received 6 December 2017, accepted 14 April 2018, published online 17 September 2018

\begin{abstract}
A line-coloring of the finite affine space $\operatorname{AG}(n, q)$ is proper if any two lines from the same color class have no point in common, and it is complete if for any two different colors $i$ and $j$ there exist two intersecting lines, one is colored by $i$ and the other is colored by $j$. The pseudoachromatic index of $\operatorname{AG}(n, q)$, denoted by $\psi^{\prime}(\operatorname{AG}(n, q))$, is the maximum number of colors in any complete line-coloring of $\operatorname{AG}(n, q)$. When the coloring is also proper, the maximum number of colors is called the achromatic index of $\operatorname{AG}(n, q)$. We prove that $\psi^{\prime}(\mathrm{AG}(n, q)) \sim q^{1.5 n-1}$ for even $n$, and that $q^{1.5(n-1)}<\psi^{\prime}(\mathrm{AG}(n, q))<q^{1.5 n-1}$ for odd $n$. Moreover, we prove that the achromatic index of $\operatorname{AG}(n, q)$ is $q^{1.5 n-1}$ for even $n$, and we provide the exact values of both indices in the planar case.

* The authors gratefully acknowledge funding from the following sources: Gabriela Araujo-Pardo was partially supported by CONACyT-México under Projects 178395, 166306, and by PAPIIT-México under Projects IN104915 and IN107218. György Kiss was partially supported by the bilateral Slovenian-Hungarian Joint Research Project, grant no. NN 114614 (in Hungary) and N1-0032 (in Slovenia), and by the Hungarian National Foundation for Scientific Research, grant no. K 124950. Christian Rubio-Montiel was partially supported by a CONACyT-México Postdoctoral fellowship, and by the National scholarship programme of the Slovak Republic. Adrián Vázquez-Ávila was partially supported by SNI of CONACyT-México.
\end{abstract}

(ㄷ) This work is licensed under https://creativecommons.org/licenses/by/4.0/ 
Keywords: Achromatic index, complete coloring, finite affine space, pseudoachromatic index. Math. Subj. Class.: 05B25, 51E15, 05C15

\section{Introduction}

This paper is motivated by the well-known combinatorial conjecture about colorings of finite linear spaces stated by Erdős, Faber and Lovász in 1972. As a starting point, we briefly recall some definitions and state the conjecture. Let $\mathbf{S}$ be a finite linear space. A line-coloring of $\mathbf{S}$ with $k$ colors is a surjective function $\varsigma$ from the lines of $\mathbf{S}$ to the set of colors $[k]=\{1, \ldots, k\}$. For short, a line-coloring with $k$ colors is called $k$-coloring. If $\varsigma: \mathbf{S} \rightarrow[k]$ is a $k$-coloring and $i \in[k]$ then the subset of lines $\varsigma^{-1}(i)$ is called the $i$-th color class of $\varsigma$. A $k$-coloring of $\mathbf{S}$ is proper if any two lines from the same color class have no point in common. The chromatic index $\chi^{\prime}(\mathbf{S})$ of $\mathbf{S}$ is the smallest $k$ for which there exists a proper $k$-coloring of $\mathbf{S}$. The Erdós-Faber-Lovász conjecture (1972) states that if a finite linear space $\mathbf{S}$ contains $v$ points then $\chi^{\prime}(\mathbf{S}) \leq v$, see $[12,13]$.

Several papers have investigated the conjecture for particular classes of linear spaces. For instance, if each line of $\mathbf{S}$ has the same number $\kappa$ of points then $\mathbf{S}$ is called a block design or a $(v, \kappa)$-design. The conjecture is still open for designs even for $\kappa=3$, however, it was proved for finite projective spaces by Beutelspacher, Jungnickel and Vanstone [8]. It is not hard to see that the conjecture is also true for the $n$-dimensional affine space $\operatorname{AG}(n, q)$ of order $q$ defined over the Galois field $\operatorname{GF}(q)$. Indeed,

$$
\chi^{\prime}(\mathrm{AG}(n, q))=\frac{q^{n}-1}{q-1} .
$$

For some related results, see for instance [6, 7].

A natural question is to determine similar, but slightly different color parameters in finite linear spaces. A $k$-coloring of $\mathbf{S}$ is complete if for each pair of different colors $i$ and $j$ there exist two intersecting lines of $\mathbf{S}$, such that one of them belongs to the $i$-th and the other one to the $j$-th color class. Observe that any proper coloring of $\mathbf{S}$ with $\chi^{\prime}(\mathbf{S})$ colors is a complete coloring. The pseudoachromatic index $\psi^{\prime}(\mathbf{S})$ of $\mathbf{S}$ is the largest $k$ such that there exists a complete $k$-coloring (not necessarily proper) of $\mathbf{S}$. When the $k$-coloring is required to be complete and proper, the parameter is called the achromatic index and it is denoted by $\alpha^{\prime}(\mathbf{S})$. Therefore, we have that

$$
\chi^{\prime}(\mathbf{S}) \leq \alpha^{\prime}(\mathbf{S}) \leq \psi^{\prime}(\mathbf{S})
$$

Several authors studied the pseudoachromatic index, see [2, 3, 4, 5, 9, 14, 15, 17]. Moreover, in $[1,10,18]$ the achromatic indices of some block designs were also estimated.

In this paper we study the pseudoachromatic and achromatic indices of finite affine spaces. In the proofs we will often use the notion of the projective closure of $\operatorname{AG}(n, q)$. This is the finite projective space $\operatorname{PG}(n, q)=\operatorname{AG}(n, q) \cup \mathcal{H}_{\infty}$, where the points of $\mathcal{H}_{\infty}$ correspond to the parallel classes of lines in $\operatorname{AG}(n, q)$. The space $\mathcal{H}_{\infty}$ is isomorphic to $\mathrm{PG}(n-1, q)$, and it is called the hyperplane at infinity. We assume that the reader is

E-mail addresses: garaujo@matem.unam.mx (Gabriela Araujo-Pardo), kissgy@cs.elte.hu (György Kiss), christian.rubio@apolo.acatlan.unam.mx (Christian Rubio-Montiel), adrian.vazquez@unaq.edu.mx (Adrián Vázquez-Ávila) 
familiar with the most important properties of affine and projective geometries. For the detailed description of these spaces we refer to [16].

The main results in the paper are proved in Sections 2 and 3. They are stated in Theorems $1.1,1.2$ and 1.3. In these theorems $v=q^{n}$ always denotes the number of points of the finite affine space $\operatorname{AG}(n, q)$.

Theorem 1.1. For all $n$ :

$$
\psi^{\prime}(\operatorname{AG}(n, q)) \leq \frac{\sqrt{v}(v-1)}{q-1}-\Theta(q \sqrt{v} / 2) .
$$

Theorem 1.2. If $n$ is even:

$$
\frac{1}{2} \cdot \frac{\sqrt{v}(v-1)}{q-1}-\Theta(\sqrt{v} / 2) \leq \psi^{\prime}(\operatorname{AG}(n, q)) .
$$

If $n$ is odd:

$$
\frac{1}{\sqrt{q}} \cdot \frac{\sqrt{v}(v-1)}{q-1}-\Theta\left(v \sqrt{v / q^{5}}\right) \leq \psi^{\prime}(\operatorname{AG}(n, q)) .
$$

Theorem 1.3. If $n$ is even:

$$
\frac{1}{3} \cdot \frac{\sqrt{v}(v-1)}{q-1}+\Theta(v / q) \leq \alpha^{\prime}(\operatorname{AG}(n, q)) .
$$

Note that when $n$ is even Theorems 1.1 and 1.2 show that $\psi^{\prime}(\operatorname{AG}(n, q))$ grows asymptotically as $\Theta\left(v^{1.5} / q\right)$, while Theorems 1.2 and 1.3 show that $\alpha^{\prime}(\operatorname{AG}(n, q))$ grows asymptotically as $\Theta\left(v^{1.5} / q\right)$. Let us remark that no similar estimates regarding the asymptotic behavior of these indices have appeared so far in the literature.

Finally, in Section 4 we determine the exact values of pseudoachromatic and achromatic indices of arbitrary (not necessarily Desarguesian) finite affine planes and we improve the previous lower bounds in dimension 3 .

\section{Upper bounds}

In this section, upper bounds for the pseudoachromatic index of $\operatorname{AG}(n, q)$ are presented when $n>2$. The following lemma is pivotal in the proof.

Lemma 2.1. Let $\mathcal{L}$ be a set of $s$ lines in $\operatorname{AG}(n, q), n>2$. Then the number of lines of $\operatorname{AG}(n, q)$ intersecting at least one element of $\mathcal{L}$ is at most

$$
q^{2}\left(s \frac{q^{n-1}-1}{q-1}-(s-1)\right) .
$$

Proof. In $\mathrm{AG}(n, q)$ there are $q\left(\frac{q^{n}-1}{q-1}-1\right)=q^{2}\left(\frac{q^{n-1}-1}{q-1}\right)$ lines intersecting any fixed line. The number of lines intersecting two lines, say $\ell_{1}$ and $\ell_{2}$, is at least $q^{2}$, because if $\ell_{1} \cap \ell_{2}=\emptyset$ then the $q^{2}$ lines joining a point of $\ell_{1}$ and a point of $\ell_{2}$ intersect both $\ell_{1}$ and $\ell_{2}$, while, if $\ell_{1} \cap \ell_{2}=\{P\}$ then the other $\frac{q^{n}-1}{q-1}-2>q^{2}$ lines through $P$ intersect both $\ell_{1}$ and $\ell_{2}$. Consequently, the number of lines intersecting at least one element of $\mathcal{L}$ is at most

$$
s q^{2}\left(\frac{q^{n-1}-1}{q-1}\right)-(s-1) q^{2} .
$$


Notice that the previous inequality is tight, since if $\mathcal{L}$ consists of $s$ parallel lines in a plane then there are exactly $q^{2}\left(s \frac{q^{n-1}-1}{q-1}-(s-1)\right)$ lines intersecting at least one element of $\mathcal{L}$.

Lemma 2.2. Let $n>2$ be an integer. Then the colorings of the finite affine space $\operatorname{AG}(n, q)$ satisfy the inequality

$$
\psi^{\prime}(\mathrm{AG}(n, q)) \leq \frac{\sqrt{4 q^{n}\left(q^{n}-1\right)\left(q^{n}-q^{2}\right)+\left(q^{2}+1\right)^{2}(q-1)^{2}}}{2(q-1)}+\frac{q^{2}+1}{2} .
$$

Proof. Consider a complete coloring which contains $\psi^{\prime}(\mathrm{AG}(n, q))$ color classes. Then the number of lines in the smallest color class is at most

$$
s=\frac{q^{n-1}\left(q^{n}-1\right)}{(q-1) \psi^{\prime}(\mathrm{AG}(n, q))} .
$$

Each of the other $\psi^{\prime}(\operatorname{AG}(n, q))-1$ color classes must contain at least one line which intersects a line from the smallest color class. Hence, by Lemma 2.1, we obtain

$$
\psi^{\prime}(\mathrm{AG}(n, q))-1 \leq q^{2}\left(s \frac{q^{n-1}-1}{q-1}-(s-1)\right) .
$$

Multiplying it by $\psi^{\prime}(\operatorname{AG}(n, q))$, we get a quadratic inequality on $\psi^{\prime}(\operatorname{AG}(n, q))$, whence the assertion follows.

We are in a position to prove our first main theorem.

Proof of Theorem 1.1. For $n>2$ a straightforward computation shows

$$
\begin{aligned}
& 4 q^{n}\left(q^{n}-1\right)\left(q^{n}-q^{2}\right)+\left(q^{2}+1\right)^{2}(q-1)^{2} \\
& \quad=\left(2 q^{\frac{n}{2}}\left(q^{n}-1\right)-q^{\frac{n}{2}}\left(q^{2}-1\right)\right)^{2}-q^{n}\left(q^{2}-1\right)^{2}+\left(q^{2}+1\right)^{2}(q-1)^{2} \\
& <\left(2 q^{\frac{n}{2}}\left(q^{n}-1\right)-q^{\frac{n}{2}}\left(q^{2}-1\right)\right)^{2}
\end{aligned}
$$

because $n>2$ implies that $q^{n}\left(q^{2}-1\right)^{2}>\left(q^{2}+1\right)^{2}(q-1)^{2}$. This together with Inequality (2.1) give

$$
\psi^{\prime}(\mathrm{AG}(n, q)) \leq q^{\frac{n}{2}}\left(\frac{q^{n}-1}{q-1}\right)-q^{\frac{n}{2}}\left(\frac{q+1}{2}\right)+\frac{q^{2}+1}{2},
$$

which proves the theorem for $n>2$. For $n=2$ the statement is clear.

\section{Lower bounds}

In this section complete colorings of $\operatorname{AG}(n, q)$ are presented. These constructions give different bounds on $\psi^{\prime}(\mathrm{AG}(n, q))$ depending on the parity of $n$. First, we prove some geometric properties of affine and projective spaces. 
Proposition 3.1. Let $n>1$ be an integer, $\Pi_{1}$ and $\Pi_{2}$ be subspaces in $\mathrm{PG}(n, q)=$ $\operatorname{AG}(n, q) \cup \mathcal{H}_{\infty}$. Let $d_{i}$ denote the dimension of $\Pi_{i}$ for $i=1,2$. Suppose that $\Pi_{1} \cap \Pi_{2} \cap \mathcal{H}_{\infty}$ is an m-dimensional subspace and $d_{1}+d_{2}=n+1+m$. Then $\Pi_{1} \cap$ $\Pi_{2} \cap \mathrm{AG}(n, q)$ is an $(m+1)$-dimensional subspace in $\mathrm{AG}(n, q)$.

In particular, $\Pi_{1} \cap \Pi_{2}$ is a single point in $\mathrm{AG}(n, q)$ when $\Pi_{1} \cap \Pi_{2} \cap \mathcal{H}_{\infty}=\emptyset$ and $d_{1}+d_{2}=n$.

Proof. Since $\Pi_{1} \cap \Pi_{2} \cap \mathcal{H}_{\infty}$ is an $m$-dimensional subspace, $\operatorname{dim}\left(\Pi_{1} \cap \Pi_{2}\right) \leq m+1$. On the other hand, the dimension formula yields

$$
\operatorname{dim}\left(\Pi_{1} \cap \Pi_{2}\right)=\operatorname{dim} \Pi_{1}+\operatorname{dim} \Pi_{v}-\operatorname{dim}\left\langle\Pi_{1}, \Pi_{2}\right\rangle \geq d_{1}+d_{2}-n=m+1 .
$$

Thus $\Pi_{1} \cap \Pi_{2}$ is an $(m+1)$-dimensional subspace in $\operatorname{PG}(n, q)$, therefore $\Pi_{1} \cap \Pi_{2} \cap$ $\mathrm{AG}(n, q)$ is an $(m+1)$-dimensional subspace in $\mathrm{AG}(n, q)$ if $m \geq 0$.

If $m=-1$, then $\Pi_{1} \cap \Pi_{2} \cap \mathcal{H}_{\infty}=\emptyset$ and $\operatorname{dim}\left(\Pi_{1} \cap \Pi_{2}\right)=0$. Hence $\Pi_{1} \cap \Pi_{2}$ is a single point in $\operatorname{AG}(n, q)$.

In the following proposition we present a partition of the points of $\mathrm{PG}(2 k, q)$ that we will call a good partition in the rest of the paper.

Proposition 3.2. Let $k \geq 1$ be an integer and $Q \in \mathrm{PG}(2 k, q)$ be an arbitrary point. The points of $\mathrm{PG}(2 k, q) \backslash\{Q\}$ can be divided into two subsets, say $\mathcal{A}$ and $\mathcal{B}$, and one can assign a subspace $S(P)$ to each point $P \in \mathcal{A} \cup \mathcal{B}$, such that the following holds true:

- $P \in S(P)$ for all points;

- $|\mathcal{A}|=q^{2}\left(\frac{q^{2 k}-1}{q^{2}-1}\right)$ and, if $A \in \mathcal{A}$ then $S(A)$ is a $k$-dimensional subspace;

- $|\mathcal{B}|=q\left(\frac{q^{2 k}-1}{q^{2}-1}\right)$ and, if $B \in \mathcal{B}$ then $S(B)$ is a $(k-1)$-dimensional subspace;

- $S(A) \cap S(B)=\emptyset$ for all $A \in \mathcal{A}$ and $B \in \mathcal{B}$.

Proof. We prove the assertion by induction on $k$. If $k=1$ then let $\left\{\ell_{0}, \ell_{1}, \ldots, \ell_{q}\right\}$ be the set of lines through $Q$. Let $\mathcal{A}$ and $\mathcal{B}$ consist of points $\operatorname{PG}(2, q) \backslash\left\{\ell_{0}\right\}$ and $\ell_{0} \backslash\{Q\}$, respectively. If $A \in \mathcal{A}$ then let $S(A)$ be the line $A Q$, if $B \in \mathcal{B}$ then let $S(B)$ be the point $B$. These sets clearly fulfill the prescribed conditions, so $\operatorname{PG}(2, q)$ admits a good partition.

Now, let us suppose that $\mathrm{PG}(2 k, q)$ admits a good partition. In $\mathrm{PG}(2 k+2, q)$ take a $2 k$-dimensional subspace $\Pi$ which contains the point $Q$. Then $\Pi$ is isomorphic to $\operatorname{PG}(2 k, q)$, hence it has a good partition $\{Q\} \cup \mathcal{A}^{\prime} \cup \mathcal{B}^{\prime}$ with assigned subspaces $S^{\prime}(P)$. Let $H_{0}, \ldots, H_{q}$ be the pencil of hyperplanes in $\mathrm{PG}(2 k+2, q)$ with carrier $\Pi$. Let $\mathcal{B}=$ $\mathcal{B}^{\prime} \cup\left(H_{0} \backslash \Pi\right)$ and $\mathcal{A}=\operatorname{PG}(2 k+2, q) \backslash(\mathcal{B} \cup\{Q\})$. Notice that $\mathcal{A}^{\prime}$ and $\mathcal{B}^{\prime}$ have the required cardinalities, because

$$
\begin{aligned}
\left|\mathcal{A}^{\prime}\right| & =\frac{q^{2 k+3}-1}{q-1}-(|\mathcal{B}|+1)=(q+1) \frac{q^{2 k+3}-1}{q^{2}-1}-q\left(\frac{q^{2 k+2}-1}{q^{2}-1}\right)-1 \\
& =q^{2}\left(\frac{q^{2 k+2}-1}{q^{2}-1}\right), \\
\left|\mathcal{B}^{\prime}\right| & =|\mathcal{B}|+\left|H_{0} \backslash \Pi\right|=q\left(\frac{q^{2 k}-1}{q^{2}-1}\right)+q^{2 k+1}=q\left(\frac{q^{2 k+2}-1}{q^{2}-1}\right) .
\end{aligned}
$$


We assign the subspaces in the following way. If $A \in \mathcal{A}^{\prime}$ then let $S(A)$ be the $(k+1)$ dimensional subspace $\left\langle S^{\prime}(A), P\right\rangle$ where $P \in \cup_{i=1}^{q} H_{i}$ is an arbitrary point, whereas, if $A \in\left(\cup_{i=1}^{q} H_{i}\right) \backslash \Pi$ then let $S(A)$ be the $(k+1)$-dimensional subspace $\left\langle A, S^{\prime}(P)\right\rangle$ where $P \in \mathcal{A}^{\prime}$ is an arbitrary point. In both cases $S(A) \subset \cup_{i=1}^{q} H_{i}$ for all $A \in \mathcal{A}$. Similarly, if $B \in \mathcal{B}^{\prime}$ then let $S(B)$ be the $k$-dimensional subspace $\left\langle S^{\prime}(B), P\right\rangle$ where $P \in H_{0}$ is an arbitrary point, whereas, if $B \in H_{0} \backslash \Pi$ then let $S(B)$ be the $k$-dimensional subspace $\left\langle B, S^{\prime}(P)\right\rangle$ where $P \in \mathcal{B}^{\prime}$ is an arbitrary point. Also here, in both cases, $S(B) \subset H_{0}$ for all $B \in \mathcal{B}$. Moreover, the assigned subspaces satisfy the intersection condition because if $A \in \mathcal{A}$ and $B \in \mathcal{B}$ are arbitrary points then

$$
S(A) \cap S(B)=\left(S(A) \cap\left(\cup_{i=1}^{q} H_{i}\right)\right) \cap\left(S(B) \cap H_{0}\right)=S^{\prime}(A) \cap S^{\prime}(B) \cap \Pi=\emptyset .
$$

Hence $\mathrm{PG}(2 k+2, q)$ also admits a good partition, and the statement is proved.

The next theorem proves Theorem 1.2 for even dimensional finite affine spaces. Notice that the lower bound depends on the parity of $q$, but its magnitude is $\frac{\sqrt{v}(v-1)}{2(q-1)}$ in both cases, where $v=q^{n}$.

Theorem 3.3. If $k>1$ then the colorings of the even dimensional affine space, $\operatorname{AG}(2 k, q)$, satisfy the inequalities

$$
\psi^{\prime}(\mathrm{AG}(2 k, q)) \geq \begin{cases}\frac{q^{k}\left(q^{2 k}-1\right)}{2(q-1)}, & \text { if } q \text { is odd } \\ \frac{q^{k}\left(q^{2 k}-q\right)}{2(q-1)}+1, & \text { if } q \text { is even. }\end{cases}
$$

Proof. The hyperplane at infinity in the projective closure of $\mathrm{AG}(2 k, q), \mathcal{H}_{\infty}$, is isomorphic to $\mathrm{PG}(2 k-1, q)$, hence it has a $(k-1)$-spread $\mathcal{S}=\left\{S^{1}, S^{2}, \ldots, S^{q^{k}+1}\right\}$. The elements of $\mathcal{S}$ are pairwise disjoint $(k-1)$-dimensional subspaces (see [16, Theorem 4.1]). Let $\left\{P_{1}^{i}, P_{2}^{i}, \ldots, P_{\left(q^{k}-1\right) /(q-1)}^{i}\right\}$ be the set of points of $S^{i}$ for $i=1,2, \ldots$, $q^{k}+1$. For a point $P \in \mathcal{H}_{\infty}$ let $S(P)$ denote the unique element of $\mathcal{S}$ that contains $P$, and $A(P)=\left\{\Pi_{P, 1}, \Pi_{P, 2}, \ldots, \Pi_{P, q^{k}}\right\}$ denote the set of the $q^{k}$ parallel $k$-dimensional subspaces of $\operatorname{AG}(2 k, q)$ whose projective closures intersect $\mathcal{H}_{\infty}$ in $S(P)$.

We define a pairing on the set of points of $\mathcal{H}_{\infty}$ which depends on the parity of $q$. On the one hand, if $q$ is odd then let $\left(P_{j}^{i}, P_{j}^{i+1}\right)$ be the pairs for $i=1,3,5, \ldots, q^{k}$ and $j=1,2, \ldots, \frac{q^{k}-1}{q-1}$. On the other hand, if $q$ is even then $\mathcal{H}_{\infty}$ has an odd number of points, thus we give the pairing on the set of points $\mathcal{H}_{\infty} \backslash\left\{P_{1}^{1}\right\}$ : let $\left(P_{j}^{i}, P_{j}^{i+1}\right)$ be the pairs for $i=4,6, \ldots, q^{k}$ and $j=1,2, \ldots, \frac{q^{k}-1}{q-1}$, and let $\left(P_{j}^{1}, P_{j}^{2}\right),\left(P_{j+1}^{2}, P_{j+1}^{3}\right),\left(P_{j+1}^{1}, P_{j}^{3}\right)$ and $\left(P_{1}^{2}, P_{1}^{3}\right)$ be the pairs for $i=1,2,3$ and $j=2,4,6, \ldots, \frac{q^{k}-1}{q-1}-1$.

Let $(U, V)$ be any pair of points. Then, by defintion, $S(U) \neq S(V)$. Let the color class $C_{U, V, i}$ contain the lines joining either $U$ and a point from $\Pi_{U, i}$, or $V$ and a point from $\Pi_{V, i}$, for $i=1,2, \ldots, q^{k}$. Clearly, $(U, V)$ defines $q^{k}$ color classes, each one consists of the parallel lines of one subspace in $A(U)$ and the parallel lines of one subspace in $A(V)$. Finally, if $q$ is even, then let the color class $C_{1}$ consist of all lines of $\operatorname{AG}(2 k, q)$ whose point at infinity is $P_{1}^{1}$.

We divided the points of $\mathcal{H}_{\infty}$ into $\frac{q^{2 k}-1}{2(q-1)}$ pairs if $q$ is odd, and into $\frac{q^{2 k}-q}{2(q-1)}$ pairs if $q$ is even. Consequently, the number of color classes is equal to $\frac{q^{2 k}-1}{2(q-1)} q^{k}$ when $q$ is odd, and it is equal to $\frac{q^{2 k}-q}{2(q-1)} q^{k}+1$ when $q$ is even. 
Now, we show that the coloring is complete. The class $C_{1}$ obviously intersects any other class. Let $C_{U, V, i}$ and $C_{W, Z, j}$ be two color classes. Then $S(U)$ and $S(V)$ are distinct elements of the spread $\mathcal{S}$ and $S(W)$ is also an element of $\mathcal{S}$. Hence we may assume, without loss of generality, that $S(U) \cap S(W)=\emptyset$. As

$$
\operatorname{dim}\left(S(U) \cup \Pi_{U, i}\right)=\operatorname{dim}\left(S(W) \cup \Pi_{W, j}\right)=k
$$

in $\mathrm{PG}(2 k, q)$, by Proposition 3.1, we have that $\Pi_{U, i} \cap \Pi_{W, j}$ consists of a single point in $\operatorname{AG}(2 k, q)$. Notice that the coloring is not proper, because the same argument shows that $\Pi_{U, i} \cap \Pi_{V, i}$ is also a single point in $\operatorname{AG}(2 k, q)$.

For odd dimensional spaces we have a slightly weaker estimate. In this case, the magnitude of the lower bound is $\frac{1}{\sqrt{q}} \cdot \frac{\sqrt{v}(v-1)}{q-1}$, where $v=q^{n}$.

Theorem 3.4. If $k \geq 1$ then the colorings of the odd dimensional affine space, $\mathrm{AG}(2 k+1, q)$, satisfy the inequality

$$
q^{k+2}\left(\frac{q^{2 k}-1}{q^{2}-1}\right)+1 \leq \psi^{\prime}(\operatorname{AG}(2 k+1, q)) .
$$

Proof. The hyperplane at infinity in the projective closure of $\mathrm{AG}(2 k+1, q), \mathcal{H}_{\infty}$, is isomorphic to $\mathrm{PG}(2 k, q)$. Hence, by Proposition $3.2, \mathcal{H}_{\infty}$ admits a good partition $\mathcal{H}_{\infty}=\mathcal{A} \cup \mathcal{B} \cup$ $\{Q\}$ with assigned subspaces $S(U)$. Let $\mathcal{A}=\left\{P_{1}, P_{2}, \ldots, P_{t}\right\}$ and $\mathcal{B}=\left\{R_{1}, R_{2}, \ldots, R_{s}\right\}$ where $t=q^{2}\left(\frac{q^{2 k}-1}{q^{2}-1}\right)$ and $s=q\left(\frac{q^{2 k}-1}{q^{2}-1}\right)$.

For a point $P_{i} \in \mathcal{A}$ let $A\left(P_{i}\right)=\left\{\Pi_{P_{i}, 1}, \Pi_{P_{i}, 2}, \ldots, \Pi_{P_{i}, q^{k}}\right\}$ denote the set of the $q^{k}$ parallel $(k+1)$-dimensional subspaces of $\mathrm{AG}(2 k+1, q)$ whose projective closures intersect $\mathcal{H}_{\infty}$ in $S\left(P_{i}\right)$. Similarly, for a point $R_{j} \in \mathcal{B}$ let $B\left(R_{j}\right)=\left\{\Pi_{R_{j}, 1}, \Pi_{R_{j}, 2}, \ldots, \Pi_{R_{j}, q^{k+1}}\right\}$ denote the set of the $q^{k+1}$ parallel $k$-dimensional subspaces of $\operatorname{AG}(2 k+1, q)$ whose projective closures intersect $\mathcal{H}_{\infty}$ in $S\left(R_{j}\right)$.

Now, we define the color classes. Let $C_{1}$ be the color class that contains all lines of $\operatorname{AG}(2 k+1, q)$ whose point at infinity is $Q$. Let the color class $C_{i, j, m}$ contain the lines joining either $P_{(j-1) q+i}$ and a point from $\Pi_{P_{(j-1) q+i}, m}$, or $R_{j}$ and a point from $\Pi_{R_{j},(i-1) q^{k}+m}$ for $j=1,2, \ldots, s, i=1,2, \ldots, q$ and $m=1,2, \ldots, q^{k}$. Counting the number of color classes of type $C_{i, j, m}$, we obtain $s \cdot q \cdot q^{k}=q^{k+2}\left(\frac{q^{2 k}-1}{q^{2}-1}\right)$. Each color class consists of the parallel lines of one subspace in $A\left(P_{(j-1) q+i}\right)$ and the parallel lines of one subspace in $B\left(R_{j}\right)$. Clearly, the total number of color classes is $1+q^{k+2}\left(\frac{q^{2 k}-1}{q^{2}-1}\right)$. The color class $C_{1}$ contains $q^{2 k}$ lines and each of the classes of type $C_{i, j, m}$ consists of $q^{k}+q^{k-1}$ lines.

To prove that the coloring is complete, notice that the class $C_{1}$ obviously intersects any other class. Let $C_{i, j, m}$ and $C_{i^{\prime}, j^{\prime}, m^{\prime}}$ be two color classes other than $C_{1}$. Consider the projective closures of those elements of $A\left(P_{(j-1) q+i}\right)$ and $B\left(R_{j^{\prime}}\right)$ whose lines are contained in $C_{i, j, m}$ and in $C_{i^{\prime}, j^{\prime}, m^{\prime}}$, respectively. One of these subspaces is a $(k+1)$ dimensional, whereas the other one is a $k$-dimensional subspace in $\operatorname{PG}(2 k+1, q)$, and they have no point in common in $\mathcal{H}_{\infty}$. Thus, by Proposition 3.1, their intersection is a single point in $\mathrm{AG}(2 k+1, q)$.

The coloring is not proper, because the same argument shows that $\Pi_{P_{(j-1) q+i}, m} \cap$ $\Pi_{R_{j},(i-1) q^{k}+m}$ is also a point in $\mathrm{AG}(2 k+1, q)$, thus $C_{i, j, m}$ contains a pair of intersecting lines. 
Now, we are ready to prove our second main theorem.

Proof of Theorem 1.2. If $n$ is even then Theorem 3.3 gives the result at once. If $n$ is odd then $v=q^{2 k+1}$, hence $\sqrt{v / q}=q^{k}$. From the estimate of Theorem 3.4 we get

$$
\begin{aligned}
q^{k+2}\left(\frac{q^{2 k}-1}{q^{2}-1}\right)+1 & =\frac{q^{3 k+2}-q^{k+2}}{q^{2}-1}+1 \\
& =\frac{(q+1)\left(q^{3 k+1}-q^{k}\right)}{q^{2}-1}-\frac{q^{3 k+1}+q^{k+2}-q^{k+1}-q^{k}}{q^{2}-1}+1 \\
& =\frac{1}{\sqrt{q}} \frac{\sqrt{v}(v-1)}{q-1}-\frac{q^{3 k+1}+q^{k+2}-q^{k+1}-q^{k}}{q^{2}-1}+1,
\end{aligned}
$$

which proves the statement.

Next, recall that a lower bound for the achromatic index require a proper and complete line-coloring of $\operatorname{AG}(n, q)$. We consider only the even dimensional case.

Theorem 3.5. Let $k>1$ and $\epsilon=0,1$ or 2 , such that $q^{k}+1 \equiv \epsilon(\bmod 3)$. Then the achromatic index of the even dimensional finite affine space $\mathrm{AG}(2 k, q)$ satisfies the inequality

$$
\left(\frac{q^{k}+1-\epsilon}{3}\left(q^{k}+2\right)+\epsilon\right) \frac{q^{k}-1}{q-1} \leq \alpha^{\prime}(\operatorname{AG}(2 k, q)) .
$$

Proof. The hyperplane at infinity in the projective closure of $\operatorname{AG}(2 k, q), \mathcal{H}_{\infty}$, is isomorphic to $\mathrm{PG}(2 k-1, q)$, hence it admits a $(k-1)$-spread $\mathcal{L}=\left\{\ell_{1}, \ell_{2}, \ldots, \ell_{q^{k}+1}\right\}$. Let $\mathcal{A}\left(\ell_{i}\right)=\left\{\Pi_{\ell_{i}, 1}, \Pi_{\ell_{i}, 2}, \ldots, \Pi_{\ell_{i}, q^{k}}\right\}$ denote the set of the $q^{k}$ parallel $k$-dimensional subspaces in $\operatorname{AG}(2 k, q)$ whose projective closures intersect $\mathcal{H}_{\infty}$ in $\ell_{i}$. Then, by Proposition 3.1, the intersection $\Pi_{\ell_{i}, s} \cap \Pi_{\ell_{j}, t}$ is a single affine point for all $i \neq j$ and $1 \leq s, t \leq q^{k}$.

First, to any triple of $(k-1)$-dimensional subspaces, $e, f, g \in \mathcal{L}$, we assign $q^{k}+2$ color classes as follows. Take a fourth $(k-1)$-dimensional subspace $d \in \mathcal{L}$, and, for $u=\left(q^{k}-1\right) /(q-1)$, denote the points of the $(k-1)$-dimensional subspaces $d, e, f$ and $g$ by $D_{1}, D_{2}, \ldots, D_{u}, E_{1}, E_{2}, \ldots, E_{u}, F_{1}, F_{2}, \ldots, F_{u}$ and $G_{1}, G_{2}, \ldots, G_{u}$, respectively. For any triple $\left(D_{i}, e, g\right)$ there is a unique line through $D_{i}$ which intersects the skew subspaces $e$ and $g$. We can choose the numbering of the points $E_{i}$ and $G_{i}$ such that the line $E_{i} G_{i}$ intersects $d$ in $D_{i}$ for $i=1,2, \ldots, u$; the numbering of the points $F_{i}$, such that the line $D_{i} F_{i+1}$ intersects $d$ and $g$ for $i=1,2, \ldots, u-1$, and, finally, choose the line $D_{u} F_{1}$ that intersects $d$ and $g$. Notice that this construction implies that the line $D_{i} F_{i}$ does not intersect $g$ for $i=1,2, \ldots, u$. Let the points of $\Pi_{d, 1}$ denote by $M_{1}, M_{2}, \ldots, M_{q^{k}}$. We can choose the numbering of the elements of $\mathcal{A}(e), \mathcal{A}(f)$ and $\mathcal{A}(g)$ such that $\Pi_{e, i} \cap \Pi_{f, i} \cap \Pi_{g, i}=\left\{M_{i}\right\}$ for $i=1,2, \ldots, q^{k}$.

We define three types of color classes for $i=1,2, \ldots, u$ and $j=1,2, \ldots, q^{k}$. Let $B_{e, f, g}^{i, 0}$ and $B_{e, f, g}^{i, 1}$ be the color classes that contain the lines through $M_{j}$ whose point at infinity is $E_{i}$ and $F_{i}$, respectively. Let $C_{e, f, g}^{i, j}$ be the color class that contains the lines in $\Pi_{e, i}$ whose point at infinity is $E_{j}$, except the line $E_{j} M_{i}$, the lines in $\Pi_{f, i}$ whose point at infinity is $F_{j}$, except the line $F_{j} M_{i}$, and the lines in $\Pi_{g, i}$ whose point at infinity is $G_{j}$. Hence each of $B_{e, f, g}^{i, 0}$ and $B_{e, f, g}^{i, 1}$ contains $q^{k}$ lines and $C_{e, f, g}^{i, j}$ contains $3 q^{k-1}-2$ lines.

Notice that for each $i \in\{1,2, \ldots, u\}$, the union of the color classes

$$
\mathcal{K}_{e, f, g}^{i}=B_{e, f, g}^{i, 0} \cup B_{e, f, g}^{i, 1} \cup_{j=1}^{q^{k}} C_{e, f, g}^{i, j}
$$


contains all lines whose point at infinity is $E_{i}, F_{i}$ or $G_{i}$. Each of the two sets of lines belonging to $B_{e, f, g}^{i, 0}$ or $B_{e, f, g}^{i, 1}$, naturally defines a $(k+1)$-dimensional subspace of $\mathrm{PG}(2 k, q)$, we denote these subspaces by $\Pi_{E_{i}}$ and $\Pi_{F_{i}}$, respectively.

For $t=0,1, \ldots,\left\lfloor\left(q^{k}-2-\epsilon\right) / 3\right\rfloor$ let $e=\ell_{3 t+1}, f=\ell_{3 t+2}, g=\ell_{3 t+3}, d=\ell_{3 t+4}$, define $\ell_{q^{k}+2-\epsilon}$ as $\ell_{1}$, and make the $q^{k}+2$ color classes $B_{e, f, g}^{i, 0}, B_{e, f, g}^{i, 1}$ and $C_{e, f, g}^{i, j}$. Finally, for each point $P$ in the subspace $\ell_{q^{k}+1}$ if $\epsilon=1$, or in $\ell_{q^{k}}$ if $\epsilon=2$, define a new color class $D^{P}$ which contains all lines whose point at infinity is $P$.

Clearly, the coloring is proper and it contains, by definition, the required number of color classes. Now, we prove that it is complete. Notice that each color class of type $D^{P}$ obviously intersects any other color class. In relation to the other cases we have that:

- The color classes $B_{\ell_{3 m+1}, \ell_{3 m+2}, \ell_{3 m+3}}^{i, j}$ and $B_{\ell_{3 m+1}, \ell_{3 m+2}, \ell_{3 m+3}}^{i^{\prime}}$ intersect, because both of them contain all points of the $k$-dimensional subspace $\prod_{\ell_{3 m+4}, 1}$.

- If $t \neq m$ then the color classes $B_{\ell_{3 t+1}, \ell_{3 t+2}, \ell_{3 t+3}}^{i, j}$ and $B_{\ell_{3 m+1}, \ell_{3 m+2}, \ell_{3 m+3}}^{i^{\prime}}$ intersect, because the $(k-1)$-dimensional subspaces $\ell_{3 t+4}$ and $\ell_{3 m+4}$ are skew in $\mathcal{H}_{\infty}$, hence the 2-dimensional intersection of the $(k+1)$-dimensional subspaces $\Pi_{E_{i}}$ or $\Pi_{F_{i}}$, according as $j=1$ or 2 , and $\Pi_{E_{i}^{\prime}}$ or $\Pi_{F_{i}^{\prime}}$, according as $j^{\prime}=1$ or 2 , is not a subspace of $\mathcal{H}_{\infty}$. Thus Proposition 3.1 implies that their intersection contains some affine points.

- The color classes $B_{\ell_{3 m+1}, \ell_{3 m+2}, \ell_{3 m+3}}^{i, j}$ and $C_{\ell_{3 t+1}, \ell_{3 t+2}, \ell_{3 t+3}}^{i^{\prime}}$ intersect in both cases $m=t$ and $m \neq t$, because the $(k-1)$-dimensional subspaces $\ell_{3 m+4}$ and $\ell_{3 t+3}$ are skew in $\mathcal{H}_{\infty}$. Again, Proposition 3.1 implies that the intersection of the $k$-dimensional subspaces $\Pi_{\ell_{3 m+4}, 1}$ (which is a subspace of either the $(k+1)$-dimensional subspace $\Pi_{E_{i}}$ or $\Pi_{F_{i}}$, according as $j=1$ or 2$)$ and $\Pi_{\ell_{3 m+3, i^{\prime}}}$ is an affine point.

- If $t \neq m$ then each pair of color classes $C_{\ell_{3 t+1}, \ell_{3 t+2}, \ell_{3 t+3}}^{i, j}$ and $C_{\ell_{3 m+1}, \ell_{3 m+2}, \ell_{3 m+3}}^{i^{\prime} j^{\prime}}$, intersects since, as previously, the $(k-1)$-dimensional subspaces $\ell_{3 t+3}$ and $\ell_{3 m+3}$ are skew in $\mathcal{H}_{\infty}$, thus Proposition 3.1 implies that the projective closures of the $k$ dimensional subspaces $\Pi_{\ell_{3 t+3}, i}$ and $\Pi_{\ell_{3 m+3}, i^{\prime}}$ intersect each other in $\operatorname{AG}(2 k, q)$.

- Finally, we prove that each pair of classes $C_{\ell_{3 t+1}, \ell_{3 t+2}, \ell_{3 t+3}}^{i, j}$ and $C_{\ell_{3 t+1}, \ell_{3 t+2}, \ell_{3 t+3}}^{i^{\prime}, j^{\prime}}$ intersects. It is obvious when $i=i^{\prime}$. Suppose that $i \neq i^{\prime}$, let $M_{i}=\Pi_{\ell_{3 t+1}, i} \cap$ $\Pi_{\ell_{3 t+2}, i} \cap \Pi_{\ell_{3 t+3}, i}$ and $M_{i^{\prime}}=\Pi_{\ell_{3 t+1}, i^{\prime}} \cap \Pi_{\ell_{3 t+2}, i^{\prime}} \cap \Pi_{\ell_{3 t+3}, i^{\prime}}$. Since the points $M_{i}$ and $M_{i^{\prime}}$ are in $\Pi_{\ell_{3 t+4}, 1}$, the line $M_{i} M_{i^{\prime}}$ intersects $\mathcal{H}_{\infty}$ in $\ell_{3 t+4}$. Take the point $T=$ $M_{i} M_{i^{\prime}} \cap \ell_{3 t+4}$ and the lines $E_{j} T$ and $F_{j} T$. Clearly, at least one of these lines does not intersect $\ell_{3 t+3}$, we may assume without loss of generality, that $E_{j} T \cap \ell_{3 t+3}=\emptyset$.

By Proposition 3.1, there exist affine points $N_{i}=\Pi_{\ell_{3 t+1}, i} \cap \Pi_{\ell_{3 t+3}, i^{\prime}}$ and $N_{i^{\prime}}=$ $\Pi_{\ell_{3 t+1}, i^{\prime}} \cap \Pi_{\ell_{3 t+3}, i}$. Suppose that $N_{i} \in E_{j^{\prime}} M_{i^{\prime}}$ and $N_{i^{\prime}} \in E_{j} M_{i}$. Then $\ell_{3 t+1} \cap$ $M_{i} M_{i^{\prime}}=\emptyset$, hence $\left\langle\ell_{3 t+1}, M_{i} M_{i^{\prime}}\right\rangle$ is a $(k+1)$-dimensional subspace $\Sigma_{k+1}$, which intersects $\mathcal{H}_{\infty}$ in a $k$-dimensional subspace $\Sigma_{k}$. Obviously, $\Sigma_{k}$ also contains the points $E_{j}$ and $E_{j^{\prime}}$. Then $\Sigma_{k}=\left\langle\ell_{3 t+1}, T\right\rangle$, and $\Sigma_{k} \cap \ell_{3 t+3}$ is a single point, say $U$. As the lines $N_{i^{\prime}} M_{i}$ and $N_{i} M_{i^{\prime}}$ are in the $k$-dimensional subspaces $\Pi_{\ell_{3 t+3}, i}$ and $\Pi_{\ell_{3 t+3}, i^{\prime}}$, respectively, there exist the points $N_{i^{\prime}} M_{i} \cap \ell_{3 t+3}$ and $N_{i} M_{i^{\prime}} \cap \ell_{3 t+3}$. Moreover, we have that $N_{i^{\prime}} M_{i} \cap \ell_{3 t+3}=N_{i} M_{i^{\prime}} \cap \ell_{3 t+3}=U$. Hence the points $N_{i}, M_{i}, N_{i^{\prime}}$ and $M_{i^{\prime}}$ are contained in a 2-dimensional subspace $\Sigma_{2}$, and $\Sigma_{2} \cap \mathcal{H}_{\infty}$ contains the points $U, E_{j}, E_{j^{\prime}}$ and $T$. Consequently, $\Sigma_{2} \cap \mathcal{H}_{\infty}$ is the line $E_{j} T$ and it contains the point $U$, thus $E_{j} T$ intersects the subspace $\ell_{3 t+3}$, contradiction. 
Thus $N_{i} \notin E_{j^{\prime}} M_{i^{\prime}}$ or $N_{i^{\prime}} \notin E_{j} M_{i}$. This implies that $N_{i}$ or $N_{i^{\prime}}$ is a common point of the color classes $C_{\ell_{3 t+1}, \ell_{3 t+2}, \ell_{3 t+3}}^{i, j}$ and $C_{\ell_{3 t+1}, \ell_{3 t+2}, \ell_{3 t+3}^{\prime}}$.

In consequence, the coloring is complete.

To conclude this section we prove our third main theorem.

Proof of Theorem 1.3. As $v=q^{2 k}$, from Theorem 3.5 we get

$$
\begin{aligned}
\left(\frac{q^{k}+1-\epsilon}{3}\left(q^{k}+2\right)+\epsilon\right) \frac{q^{k}-1}{q-1} & =\frac{q^{3 k}+(2-\epsilon) q^{2 k}+(2 \epsilon-1) q^{k}-2-\epsilon}{3(q-1)} \\
& =\frac{1}{3} \frac{\sqrt{v}(v-1)}{q-1}+\frac{(2-\epsilon) v+2 \epsilon \sqrt{v}-2-\epsilon}{3(q-1)},
\end{aligned}
$$

which proves the statement.

\section{Small dimensions}

In this section, we improve on our bounds in two and three dimensions. First, we prove the exact values of achromatic and pseudoachromatic indices of finite affine planes. Due to the fact that there exist non-desarguesian affine planes, we use the notation $\mathrm{A}_{q}$ for an arbitrary affine plane of order $q$. For the axiomatic definition of $\mathrm{A}_{q}$ we refer to [11]. The basic combinatorial properties of $\mathrm{A}_{q}$ are the same as of $\mathrm{AG}(2, q)$.

Theorem 4.1. Let $\mathrm{A}_{q}$ be any affine plane of order $q$. Then

$$
\chi^{\prime}\left(\mathrm{A}_{q}\right)=\alpha^{\prime}\left(\mathrm{A}_{q}\right)=q+1 \text {. }
$$

Proof. Let $\mathscr{S}_{1}, \mathscr{S}_{2}, \ldots, \mathscr{S}_{q+1}$ denote the $q+1$ parallell classes of lines in $\mathrm{A}_{q}$. Two lines have a point in common if and only if they belong to distinct parallel classes. Hence, if we define a coloring $\phi$ with $q+1$ colors such that a line $\ell$ gets color $i$ if and only if $\ell \in \mathscr{S}_{i}$ then $\phi$ is proper, so $q+1 \leq \chi^{\prime}\left(\mathrm{A}_{q}\right)$.

Since $\chi^{\prime}\left(\mathrm{A}_{q}\right) \leq \alpha^{\prime}\left(\mathrm{A}_{q}\right)$, it is enough to prove that $\alpha^{\prime}\left(\mathrm{A}_{q}\right) \leq q+1$. Suppose to the contrary that $\psi$ is a complete and proper coloring with $m>q+1$ color classes. As $\psi$ is proper, each color class must be a subset of a parallel class. By the pigeonhole principle, $m>q+1$ implies that there exist at least two color classes that are subsets of the same parallel class. Hence they do not contain intersecting lines, contradicting to the completeness of $\psi$. Thus $\alpha^{\prime}\left(\mathrm{A}_{q}\right) \leq q+1$, the theorem is proved.

Theorem 4.2. Let $\mathrm{A}_{q}$ be any affine plane of order $q$. Then

$$
\psi^{\prime}\left(\mathrm{A}_{q}\right)=\left\lfloor\frac{(q+1)^{2}}{2}\right\rfloor
$$

Proof. First, we prove that $\psi^{\prime}\left(\mathrm{A}_{q}\right) \leq\left\lfloor\frac{(q+1)^{2}}{2}\right\rfloor$. Suppose to the contrary that $\varphi$ is a complete coloring of $\mathrm{A}_{q}$ with $\left\lfloor\frac{(q+1)^{2}}{2}\right\rfloor+1$ color classes. As $\mathrm{A}_{q}$ has $q^{2}+q$ lines, this implies that $\varphi$ has at most $q^{2}+q-\left(\left\lfloor\frac{(q+1)^{2}}{2}\right\rfloor+1\right)$ color classes of cardinality greater than one. Thus, there are at least

$$
\left\lfloor\frac{(q+1)^{2}}{2}\right\rfloor+1-\left(q^{2}+q-\left(\left\lfloor\frac{(q+1)^{2}}{2}\right\rfloor+1\right)\right)= \begin{cases}q+2, & \text { if } q \text { is even } \\ q+3, & \text { if } q \text { is odd }\end{cases}
$$


color classes of size one. Hence, again by the pigeonhole principle, there are at least two color classes of size one belonging to the same parallel class. They have empty intersection, so $\varphi$ is not complete. This contradiction shows that $\psi^{\prime}\left(\mathrm{A}_{q}\right) \leq\left\lfloor\frac{(q+1)^{2}}{2}\right\rfloor$.

We go on to give a complete coloring of $\mathrm{A}_{q}$ with $\left\lfloor\frac{(q+1)^{2}}{2}\right\rfloor$ color classes. Let $P$ be a point and $e_{1}, e_{2}, \ldots, e_{q+1}$ be the lines through $P$. For $i=1,2, \ldots, q+1$ let $\mathscr{S}_{i}$ be the parallel class containing $e_{i}$ and denote the $q-1$ lines in the set $\mathscr{S}_{i} \backslash\left\{e_{i}\right\}$ by $\ell_{i}, \ell_{(q+1)+i}, \ldots, \ell_{(q-2)(q+1)+i}$. Then:

$$
\bigcup_{i=1}^{q}\left(\mathscr{S}_{i} \backslash\left\{e_{i}\right\}\right)=\left\{\ell_{1}, \ell_{2}, \ldots, \ell_{q^{2}-1}\right\},
$$

and $\ell_{j}$ and $\ell_{j+1}$ are non-parallel lines for all $1 \leq j<q^{2}-1$. For better clarity, we construct $q+1$ color classes with even indices and $\left|\frac{q^{2}-1}{2}\right|$ color classes with odd indices. Let the color class $C_{2 k}$ consist of one line, $e_{k}$, for $k=1,2, \ldots, q+1$. Let the color class $C_{2 k-1}$ contain the lines $\ell_{2 k-1}$ and $\ell_{2 k}$ for $k=1,2, \ldots,\left\lfloor\frac{q^{2}-1}{2}\right\rfloor$, finally, if $q$ is even, let the color class $C_{q^{2}-3}$ contain the line $\ell_{q^{2}-1}$, too.

The coloring is complete, because color classes having even indices intersect at $P$, and each color class with odd index contains two non-parallel lines whose union intersects all lines of the plane.

Our last construction gives a lower bound for the achromatic index of $\operatorname{AG}(3, q)$. As $\alpha^{\prime}(\mathrm{AG}(3, q)) \leq \psi^{\prime}(\mathrm{AG}(3, q))$, this can be considered as well as a lower estimate on the pseudoachromatic index of $\operatorname{AG}(3, q)$ and this bound is better than the general one proved in Theorem 3.4. We use the cyclic model of $\operatorname{PG}(2, q)$ to make the coloring. The detailed description of this model can be found in [16, Theorem 4.8 and Corollary 4.9]. We collect the most important properties of the cyclic model in the following proposition.

Proposition 4.3. Let $q$ be a prime power. Then the group $\mathbb{Z}_{q^{2}+q+1}$ admits a perfect difference set $D=\left\{d_{0}, d_{1}, d_{2}, \ldots, d_{q}\right\}$, that is the $q^{2}+q$ integers $d_{i}-d_{j}(i \neq j)$ are all distinct modulo $q^{2}+q+1$. We may assume without loss of generality that $d_{0}=0$ and $d_{1}=1$. The plane $\operatorname{PG}(2, q)$ can be represented in the following way. The points are the elements of $\mathbb{Z}_{q^{2}+q+1}$, the lines are the subsets

$$
D+j=\left\{d_{i}+j: d_{i} \in D\right\}
$$

for $j=0,1, \ldots, q^{2}+q$, and the incidence is the set theoretical inclusion.

Theorem 4.4. The achromatic index of $\mathrm{AG}(3, q)$ satisfies the inequality:

$$
\frac{q(q+1)^{2}}{2}+1 \leq \alpha^{\prime}(\mathrm{AG}(3, q)) .
$$

Proof. The plane at infinity in the projective closure of $\mathrm{AG}(3, q), \mathcal{H}_{\infty}$, is isomorphic to $\operatorname{PG}(2, q)$, hence it has a cyclic representation (described in Proposition 4.3). Let $v=q^{2}+$ $q+1$, let the points and the lines of $\mathcal{H}_{\infty}$ be $P_{1}, P_{2}, \ldots, P_{v}$, and $\ell_{1}, \ell_{2}, \ldots, \ell_{v}$, respectively. We can choose the numbering such that for $i=1,2,3, \ldots, v$ the line $\ell_{i}$ contains the points $P_{i}, P_{i+1}$ and $P_{i-d}$ (where $0 \neq d \neq 1$ is a fixed element of the difference set $D$, and the subscripts are taken modulo $v$ ). 
Let $\mathcal{A}\left(P_{i}\right)=\left\{\Pi_{P_{i}, 1}, \Pi_{P_{i}, 2}, \ldots, \Pi_{P_{i}, q}\right\}$ denote the set of the $q$ parallel planes in $\operatorname{AG}(3, q)$ whose projective closures intersect $\mathcal{H}_{\infty}$ in $\ell_{i}$, and $\overline{\Pi_{P_{i}, j}}$ denote the projective closure of $\Pi_{P_{i}, j}$ for $i=1,3, \ldots, v$, and $j=1,2, \ldots, q$. Let $W_{i}$ be a plane whose projective closure intersects $\mathcal{H}_{\infty}$ in $\ell_{i-d}$. Then the projective closure of each element of $A\left(P_{i}\right) \cup A\left(P_{i+1}\right)$ intersects $W_{i}$ in a line whose point at infinity is $P_{i}$, so we can choose the numbering of the elements of $A\left(P_{i}\right)$ and $A\left(P_{i+1}\right)$, such that $\overline{\Pi_{P_{i}, j}} \cap \overline{\Pi_{P_{i+1}, j}} \subset W_{i}$ for $i=1,3, \ldots, v-2$, and $j=1,2, \ldots, q$. Let $e_{j}^{i}$ denote the line $\Pi_{P_{i}, j} \cap \Pi_{P_{i+1}, j}$.

We assign $q+1$ color classes to the pair $\left(P_{i}, P_{i+1}\right)$ for $i=1,3, \ldots, v-2$. Let the color class $C_{0}^{i}$ contain the lines $e_{1}^{i}, e_{2}^{i} \ldots, e_{q}^{i}$. For $j=1,2, \ldots, q$, let the color class $C_{j}^{i}$ contain those lines of $\Pi_{P_{i}, j}$ whose point at infinity is $P_{i}$, except the line $e_{j}^{i}$, and the $q$ parallel lines of $\Pi_{P_{i+1}, j}$ whose point at infinity is $P_{i+1}$. Finally, let the color class $C^{v}$ contain all lines whose point at infinity is $P_{v}$. In this way we constructed

$$
(q+1) \frac{v-1}{2}+1=\frac{q(q+1)^{2}}{2}+1
$$

color classes and each line belongs to exactly one of them, because $C_{0}^{i}$ contains $q$ lines, $C_{j}^{i}$ contains $2 q-1$ lines for each $j=1,2, \ldots, q$. and $C^{v}$ contains $q^{2}$ lines.

The coloring is proper by construction. The color class $C^{v}$ obviously intersects any other class. For other pairs of color classes, two major cases are distinguished when we prove the completeness. On the one hand, if $i \neq k$ then we have:

- $C_{0}^{i} \cap C_{0}^{k} \neq \emptyset$, because the planes $W_{i}$ and $W_{k}$ intersect each other;

- if $j>0$ then $C_{0}^{i} \cap C_{j}^{k} \neq \emptyset$, because the planes $W_{i}$ and $\Pi_{P_{k+1}, j}$ intersect each other;

- if $m>0$ and $j>0$ then $C_{m}^{i} \cap C_{j}^{k} \neq \emptyset$, because the planes $\Pi_{P_{i+1}, m}$ and $\Pi_{P_{k+1}, j}$ intersect each other.

On the other hand, color classes having the same superscript also have non-empty intersection:

- $C_{0}^{i} \cap C_{j}^{i} \neq \emptyset$, because the planes $W_{i}$ and $\Pi_{P_{i+1}, j}$ intersect each other;

- if $j \neq k$ then the planes $\Pi_{P_{i}, j}$ and $\Pi_{P_{i+1}, k}$ intersect in a line $f$ and $f \neq e_{j}^{i}$, hence its points are not removed from $\Pi_{P_{i}, j}$, so $C_{j}^{i} \cap C_{k}^{i} \neq \emptyset$.

Hence the coloring is also complete, this proves the theorem.

\section{References}

[1] G. Araujo-Pardo, Gy. Kiss, C. Rubio-Montiel and A. Vázquez-Ávila, On line colorings of finite projective spaces, 2018, arXiv:1702.06769 [math.CO].

[2] G. Araujo-Pardo, J. J. Montellano-Ballesteros, C. Rubio-Montiel and R. Strausz, On the pseudoachromatic index of the complete graph II, Bol. Soc. Mat. Mex. 20 (2014), 17-28, doi: 10.1007/s40590-014-0007-9.

[3] G. Araujo-Pardo, J. J. Montellano-Ballesteros, C. Rubio-Montiel and R. Strausz, On the pseudoachromatic index of the complete graph III, Graphs Combin. 34 (2018), 277-287, doi: 10.1007/s00373-017-1872-6.

[4] G. Araujo-Pardo, J. J. Montellano-Ballesteros and R. Strausz, On the pseudoachromatic index of the complete graph, J. Graph Theory 66 (2011), 89-97, doi:10.1002/jgt.20491. 
[5] G. Araujo-Pardo and C. Rubio-Montiel, Pseudoachromatic and connected-pseudoachromatic indices of the complete graph, Discrete Appl. Math. 231 (2017), 60-66, doi:10.1016/j.dam. 2017.03.019.

[6] G. Araujo-Pardo, C. Rubio-Montiel and A. Vázquez-Ávila, Note on the Erdős-Faber-Lovász conjecture: quasigroups and complete digraphs, Ars Combin., in press.

[7] G. Araujo-Pardo and A. Vázquez-Ávila, A note on Erdős-Faber-Lovász conjecture and edge coloring of complete graphs, Ars Combin. 129 (2016), 287-298.

[8] A. Beutelspacher, D. Jungnickel and S. A. Vanstone, On the chromatic index of a finite projective space, Geom. Dedicata 32 (1989), 313-318, doi:10.1007/bf00147923.

[9] A. Bouchet, Indice achromatique des graphes multiparti complets et réguliers, Cahiers Centre Études Rech. Opér. 20 (1978), 331-340.

[10] C. J. Colbourn and M. J. Colbourn, Greedy colourings of Steiner triple systems, in: A. Barlotti, P. V. Ceccherini and G. Tallini (eds.), Combinatorics '81, North-Holland, Amsterdam, volume 18 of Annals of Discrete Mathematics, pp. 201-207, 1983, proceedings of the International Conference on Combinatorial Geometries and their Applications held in Rome, June 7 $12,1981$.

[11] P. Dembowski, Finite Geometries, Classics in Mathematics, Springer-Verlag, Berlin, 1997, doi:10.1007/978-3-642-62012-6, reprint of the 1968 original.

[12] P. Erdős, Problems and results in combinatorial analysis and combinatorial analysis, in: C. St. J. A. Nash-Williams and J. Sheehan (eds.), Proceedings of the Fifth British Combinatorial Conference, Utilitas Mathematica Publishing, Winnipeg, Manitoba, volume 15 of Congressus Numerantium, pp. 169-192, 1976, held at the University of Aberdeen, Aberdeen, July $14-18$, 1975.

[13] P. Erdôs, On the combinatorial problems which I would most like to see solved, Combinatorica 1 (1981), 25-42, doi:10.1007/bf02579174.

[14] R. P. Gupta, Bounds on the chromatic and achromatic numbers of complementary graphs, in: W. T. Tutte (ed.), Recent Progress in Combinatorics, Academic Press, New York, pp. 229-235, 1969, proceedings of the Third Waterloo Conference on Combinatorics, May 1968.

[15] F. Harary, S. Hedetniemi and G. Prins, An interpolation theorem for graphical homomorphisms, Portugal. Math. 26 (1967), 453-462, http: / / eudml.org/doc/115047.

[16] J. W. P. Hirschfeld, Projective Geometries over Finite Fields, Oxford Mathematical Monographs, The Clarendon Press, New York, 2nd edition, 1998.

[17] R. E. Jamison, On the edge achromatic numbers of complete graphs, Discrete Math. 74 (1989), 99-115, doi:10.1016/0012-365x(89)90202-1.

[18] A. Rosa and C. J. Colbourn, Colorings of block designs, in: J. H. Dinitz and D. R. Stinson (eds.), Contemporary Design Theory, John Wiley \& Sons, New York, Wiley-Interscience Series in Discrete Mathematics and Optimization, pp. 401-430, 1992. 